\title{
Large-spot subthreshold transpupillary thermotherapy for chronic serous macular detachment
}

This article was published in the following Dove Press journal:

Clinical Ophthalmology

I I March 201 I

Number of times this article has been viewed

\author{
Giuseppe Lo Giudice' \\ Valentina de Belvis ${ }^{2}$ \\ Marco Tavolato' \\ Alessandro Galan' \\ 'San Paolo Ophthalmic Center, \\ San Antonio Hospital, Padova, Italy; \\ ${ }^{2}$ Paediatric Low Vision Center, \\ Paediatric Rare Eye Disease Center, \\ Department of Paediatrics, \\ University of Padova, Italy
}

Purpose: To report the effect of subthreshold transpupillary thermotherapy (TTT) in treating serous detachment of the neurosensory retina secondary to chronic central serous chorioretinopathy (CCSC).

Methods: Seven eyes from five patients with CCSC, persistent serous detachment of the neurosensory retina and a clinical course of between 12 and 60 months were treated. All eyes received large-spot TTT guided by indocyanine green angiography (ICGA). Subthreshold TTT was performed using an $810 \mathrm{~nm}$ diode laser with a spot size of $3.0 \mathrm{~mm}$ (power was set at $350 \mathrm{~mW}$ ). Treatment was applied for 60 seconds to the areas of choroidal hyperfluorescence on ICGA.

Results: The mean number of TTT sessions was $1.4 \pm 0.5$. All eyes were followed up for at least 6 months (mean 9.6 \pm 3.2 standard deviation; range 6-12 months). The mean logarithm of the minimum angle of resolution best-corrected visual acuity was significantly better compared with baseline. All TTT-treated eyes had stable or improved vision $(P<0.001)$. Mean optical coherence tomography (OCT) central foveal thickness was significantly lower in all patients $(P<0.001)$ compared with pretreatment OCT, with a reduction in subretinal fluid and resolution of serous detachment associated with anatomical fovea restoration. No patient had any treatmentrelated side effects.

Conclusion: Modified subthreshold TTT appears to have a beneficial effect in treating patients with CCSC and persistent neurosensory detachment. The encouraging results and lack of visually significant complications suggest that further investigation is warranted.

Keywords: central serous chorioretinopathy, indocyanine green angiography, neurosensory detachment, transpupillary thermotherapy

\section{Introduction}

Chronic central serous chorioretinopathy (CCSC) is a rare, severe disorder, characterized by long-term persistence of subretinal exudation, extensive areas of retinal pigment epithelium (RPE) atrophy, cystoid macular edema, choroidal neovascularization (CNV), and impairment of visual acuity (VA). ${ }^{1-5}$ Evidence is typically found of uneven and diffuse hyperfluorescence, often displaying multiple leaks with fluorescein angiography (FA), and diffuse or multifocal areas of choroidal hyperfluorescence on indocyanine green angiography (ICGA). ${ }^{6}$ Long-term follow-up studies of patients with typical idiopathic CSC have demonstrated that patients with chronic, recurrent detachment and severe visual loss are part of the spectrum of CSC, although the precise pathogenesis remains obscure..$^{7-9}$

There is still no established therapy for CCSC, and medical treatments have no proven influence on the course of the disease. ${ }^{10-12}$ Although selected cases of acute
Correspondence: Giuseppe Lo Giudice San Paolo Ophthalmic Center,

San Antonio Hospital, Via Facciolati 7I, 35100 Padua, Italy

$\mathrm{Tel}+39492050153$

Fax +394920 50I53

Email gvofta@libero.it 
CSC benefit from retinal laser photocoagulation, ${ }^{13,14}$ there is no standard treatment for CCSC. The area affected by the leakage in CCSC is often so diffuse that it requires extensive laser application, with subsequent development of bothersome scotomas. Anatomic improvements with some functional success in the treatment of CCSC have been observed for the use of photodynamic therapy (PDT) with verteporfin. ${ }^{15-17}$ The use of PDT in the treatment of chronic CCSC has not been without complication, and RPE atrophy and juxtafoveal CNV have been reported after PDT. ${ }^{18,19}$ Transpupillary thermotherapy (TTT) has been advocated in the management of macular lesions, particularly in the treatment of CNV, ${ }^{20}$ choroidal melanoma, ${ }^{21,22}$ choroidal hemangioma, ${ }^{23}$ and CSC. ${ }^{24,25}$ The advantages of diode laser $(810 \mathrm{~nm})$ in the treatment of macular disease are related to low absorption by xanthophyll and poor absorption by hemoglobin (allowing treatment through preretinal and subretinal blood), with low energy delivery, thereby avoiding damaging photoreceptor cells.

In this small pilot study, we evaluate the use of ICGAguided large-spot subthreshold TTT in the management of serous macular detachment in the CCSC.

\section{Methods}

Seven eyes from five patients with chronic CCSC were studied between February 2008 and January 2009. All patients complained of visual disturbance, metamorphopsia, and relative central scotomas. Their ocular history was significant for periodic attacks of CSC, which left some subjective visual defects. CCSC was defined in patients with persistent serous macular detachment ( $\geq 6$ months' duration; clinical course ranging between 1 and 5 years); associated RPE window defects and multiple leaks or leaks of undetermined source on FA; presence of choroidal hyperpermeability/ hyperfluorescence on ICGA; and neurosensory detachment of the macula. Pretreatment VA ranged from 20/70 to 20/500. At baseline, all patients underwent ophthalmic examination including refraction, best-corrected visual acuity (BCVA) (Snellen chart), FA, ICGA, and optical coherence tomography (OCT 3000, Humphrey Instruments, San Leandro, CA, USA). FA was performed to identify the presence of acute RPE leaks and areas of early or late hyperfluorescence, indicating diffuse decompensation of the posterior blood-retina barrier or RPE atrophy. ICGA was used to identify areas of inner choroidal vascular hyperpermeability, confirm the diagnosis, guide the TTT treatment, and rule out the possibility that the choroidal new vessel could be masquerading as CSC. OCT, centered on the foveal region, was performed using the default setting of the crosshair mode, which scans $5.0 \mathrm{~mm}$ vertically and horizontally. TTT with an $810 \mathrm{~nm}$ diode laser (Iris Medical OcuLight Slx, Iridex Corporation, Mountain, View, CA, USA) was delivered through a dedicated slit-lamp-mounted delivery system. A three-mirror Goldmann lens was used with a standard $3 \mathrm{~mm}$ spot in the laser slit lamp, producing a large-spot size of $3.24 \mathrm{~mm}$ in diameter on the retina. Treatment was applied at least 10 days after angiographic examination in all patients, with the same laser spot size and duration ( $3 \mathrm{~mm}$; 60 seconds) in all patients (the power was set at $350 \mathrm{~mW}$ ). Low-intensity TTT produces approximately $60 \%$ lower energy levels than treatment with a $3 \mathrm{~mm}$ spot size and $800 \mathrm{~mW}$ setting. One or more consecutive spots were applied to cover the area of choroidal hyperpermeability and diffuse leakage detected on ICGA. Only angiographic areas judged to be responsible for the macular detachment were selected for treatment. Treatment of the central macula was preferentially avoided. Nonetheless, treatment of the central area was performed if the foveal region was included in the affected zone. Visible retinal whitening was carefully avoided in view of subretinal exudation, shallow serous retinal detachment, presence of RPE anomalies (atrophy), and heavily pigmented fundus, which would also cause variations in temperature rise across the TTT field. All patients were scheduled for 1, 2, 3, and 6 months' follow-up after TTT. A repeat TTT was performed if angiographic dye leakage and OCT subretinal fluid persisted 3 months after treatment. The same treatment criteria as above were followed for retreatment. The study adhered to the tenets of the Declaration of Helsinki for research involving human subjects and was approved by the Ethics Committee. Written consent was obtained from all patients prior to treatment.

\section{Statistical analysis}

Clinical data were analyzed to evaluate improvement in VA and the height of the foveal detachment after treatment. BCVA was converted to logarithm of the minimum angle of resolution $(\log$ MAR) for the analysis. Analysis of variance for repeated measures was performed. The level of statistical significance was set at $P$ values lower than 0.05 .

\section{Results}

Demographic data on the patients pretreatment and post-treatment are shown in Table 1. The patient ages at the time of therapy ranged from 55 years to 69 years $(61.6 \pm 5.6$ years, mean \pm standard deviation $[S D])$. There were three men and two women. All patients had a history of over 1 year $(33.6 \pm 17.7$ months, mean $\pm \mathrm{SD}$; 


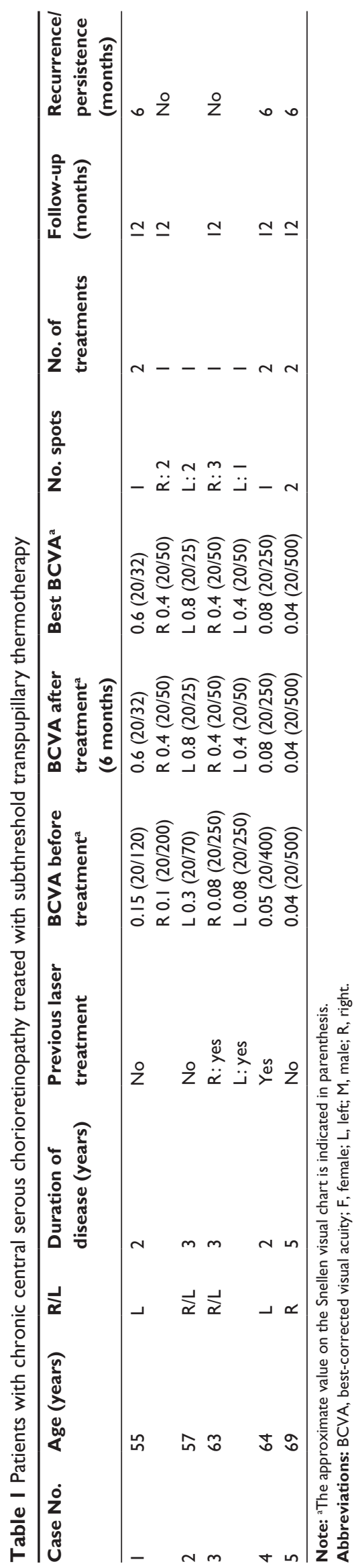

range 12-60 months) of CCSC. The follow-up period ranged from 6 months to 12 months ( $9.6 \pm 3.2$ months, mean $\pm \mathrm{SD})$. Initial VA ranged from 0.04 to $0.3(0.1 \pm 0.08 \log$ MAR, mean $\pm \mathrm{SD}$ ). Two patients had previously been treated with laser photocoagulation for acute RPE leaks. FA showed RPE window defects (all patients had some degree of transmission hyperfluorescence corresponding to RPE atrophy involving the fovea in five eyes), and subfoveal leak was identified as the cause of the macular detachments in one eye. ICG angiography revealed patches of hyperfluorescence with irregular RPE detachments and relative hypofluorescence and large areas of irregular intense/intermediate choroidal hyperpermeability in all patients. OCT showed subclinical $(<100 \mu \mathrm{m})$ or serous retinal detachment in all patients. Three patients (three eyes) had cystic changes as determined by OCT evaluations. Two patients (three eyes) had TTT involving the foveal area. Three eyes had two spots of TTT, three had one, and one had three. Four eyes of two patients with bilateral CCSC were treated in a paired manner (one eye had one spot of TTT, and three had two). Following treatment, there was complete resolution of subfoveal neurosensory detachments and restoration of foveal anatomy on OCT in four of seven eyes at 1 month (Figure 1).

One eye showed a decrease in the height of the neurosensory elevation, and two eyes did not show any sign of resolution. At months 3 and 6, the foveal detachment completely resolved in three of the five patients, corresponding to five of seven study eyes $(P=<0.001)$. Foveal detachment decreased but remained unresolved in one eye, and one eye did not show any sign of change (Figure 2).

Following treatment, there was a significant difference in VA at the 1-month, 3-month, and 6-month follow-up compared with pretreatment. VA improved $(P=<0.001)$ by two or more lines in six eyes (four of five patients). In one eye, there was persisting serous elevation with no improvement in VA. No patient had a decrease in BCVA of three or more lines during the follow-up. One eye experienced recurrent macular detachment 6 months after treatment, despite previous resolution of the detachment. After treatment, no significant vascular effects were seen on FA or ICGA at initial examination, no dark zones or perfusion defects in the choriocapillaris were seen on FA and ICGA during the follow-up, and no RPE atrophy or retinal scars were observed in the area covered by the laser spot during the follow-up. No patient had any complications from the treatment. No foveal whitening was observed in any eye during or after TTT. No large foveal atrophy was present on angiographic or thickness studies. 

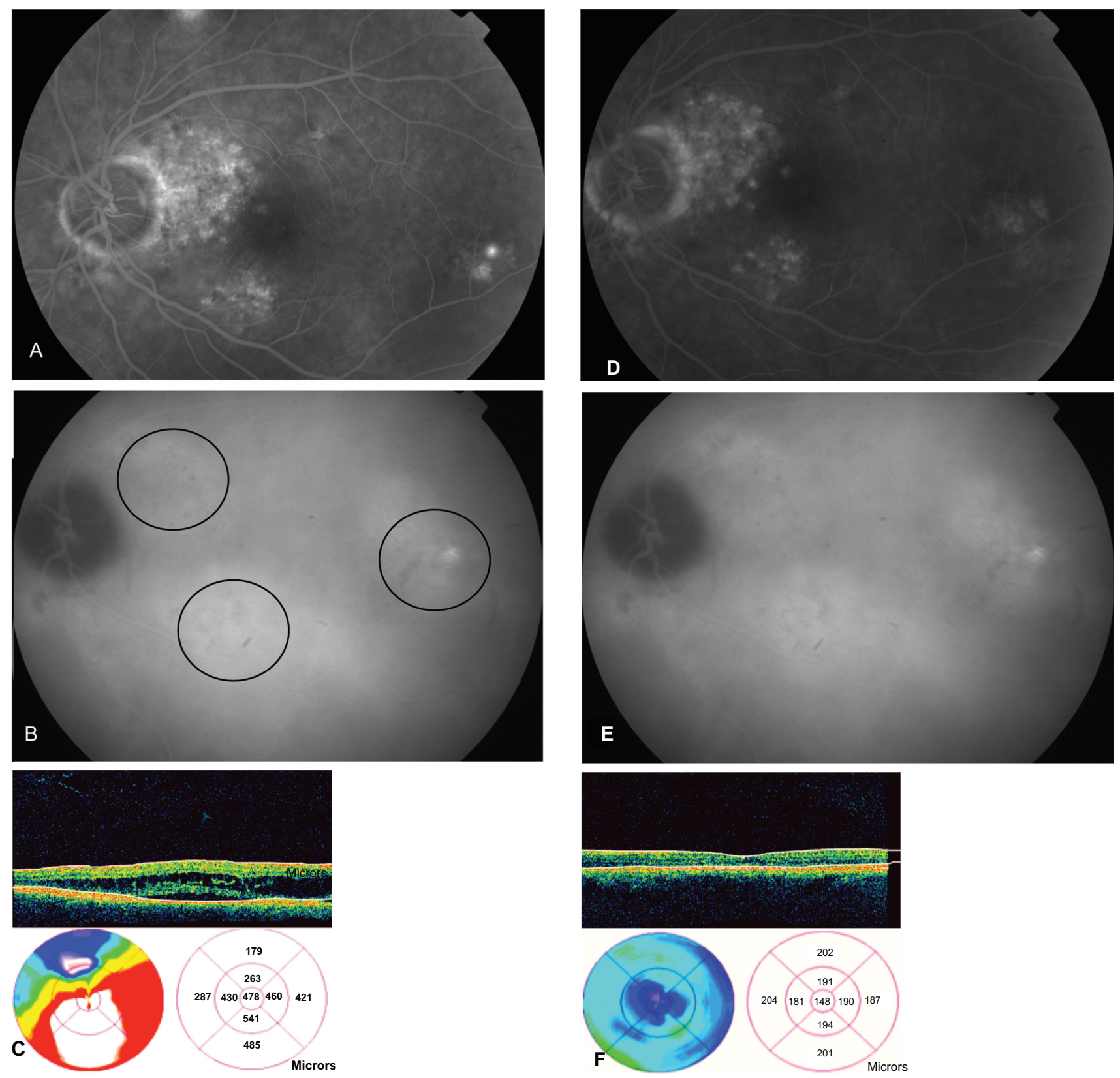

Figure I A) Fluorescein angiography (FA) shows multiple focal areas of hyperfluorescence with underlying retinal pigment epithelium (RPE) window defect involving the macula and the peripapillary region before transpupillary thermotherapy (TTT). B) Indocyanine green angiography (ICGA) showing multiple irregular patches of hyperfluorescence before TTT. Black circles indicate the area covered by large-spot subthreshold TTT. C) Optical coherence tomography (OCT) image of line scan through the macula showing macular neurosensory detachment with cystoid macular changes. D) FA showing slow, indistinct staining with absence of active focal leakage after TTT. E) ICGA showing choroidal patch of hyperfluorescence without significant vascular effects, dark zone, or perfusion defects in the choriocapillaris. OCT image showing resolution of the serous detachment and cystoid macular edema after treatment. F) No RPE atrophy or retinal scars were observed in the area covered by the laser spot during the follow-up.

\section{Discussion}

The primary tissue site effect and the cause of CCSC remain controversial. The natural course of the disease leads to progressive deterioration in VA and visual field damage due to RPE and photoreceptor degeneration. Progressive atrophic degeneration of the RPE in a multizoned distribution evolves in both persistent and recurrent detachment, leading to chronic alteration of the posterior blood-retinal barrier. Some authors consider the RPE to be the site of disturbance, with both focal and diffuse RPE cell impairment. ${ }^{26,27}$ For serous retinal detachment to form and be maintained, there must be an alteration in the net balance of fluid flow into the subretinal space. The RPE has a tremendous capacity to remove fluid from the subretinal space, and a focal leak in the RPE may produce a 


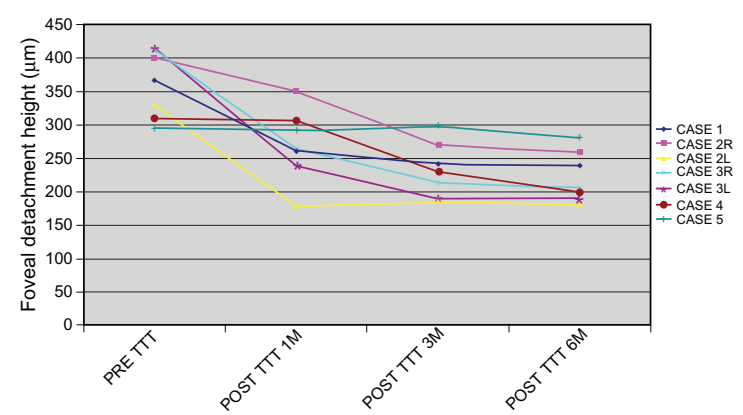

Figure $\mathbf{2}$ The time course of the height of the foveal detachment in all study eyes. The height was measured by optical coherence tomography. In six cases, the foveal detachment decreased after transpupillary thermotherapy (TTT). The height was significantly lower at 3 and 6 months after treatment $(P<0.0$ I $)$.

serous retinal detachment. Several studies relying on ICGA have suggested that the etiology lies in the choriocapillaris, with accumulation of exudates in the inner choroids, leading to serous leakage beneath the neurosensory retina and serous pigment epithelium detachment formation. ${ }^{2,6,7,28}$ There is no standard treatment for CCSC. Several reports have described focal laser photocoagulation to treat subfoveal/juxtafoveal leakage as the first approach to chronic CSC. ${ }^{13,14}$ Although laser may shorten the duration of symptoms, it does not have any impact on final vision or on the recurrence rate, with limited efficacy, because it is targeting only the RPE leak without specifically treating the underlying primary choroidal congestion and hyperpermeability or the RPE decompensation. PDT has recently been shown to have a beneficial effect in patients with CCSC, displaying a significant, rapid, short-term improvement in VA. ${ }^{15-17}$ However, several side effects occurring after PDT seem to be potentially important drawbacks in treating chronic CSC patients. ${ }^{18,19}$

In the present study, we adopted large-spot subthreshold TTT to apply a single treatment approach to the management of serous macula detachment in patients with CCSC. Five out of seven eyes ( $71 \%$ ) displayed complete resolution of serous macular elevation, and VA improved by over two or more lines during follow-up in six eyes. In parallel with the VA data, there was also a statistically significant improvement in OCT-measured macular thickness at 6 months in six eyes (85\%). The power setting was crucial for the successful treatment of TTT in our study. There is controversy about the clinical safety of TTT, limiting the possibility of applying an optimal dose of TTT. Clinical experience with TTT for CNV suggests that it is important to use subthreshold power (ie, biomicroscopically invisible laser effects) to avoid damaging the overlying neural retina, but the current treatment regimen remains largely empiric. ${ }^{30}$ In our study, the laser setting was determined by virtue of the fact that the target tissue was nonproliferative choroids. The purpose of a subthreshold power level was to avoid any associated damage to the RPE/choriocapillary complex. The spot size was dependent primarily on the location and extent of choroidal hyperpermeability on ICGA. During long exposure with a large retinal spot, the power required to produce a particular rise in retinal temperature is proportional to the diameter rather than to the area of the spot on the retina. ${ }^{31}$ Conventional TTT protocols recommend a power of $800 \mathrm{~mW}$ with a retinal spot of $3.0 \mathrm{~mm}$, giving a power/diameter ratio of $247 \mathrm{~mW} / \mathrm{mm}$. We delivered a power/diameter ratio of $108 \mathrm{~mW} / \mathrm{mm}$, which is $\approx 40 \%$ of that used in conventional TTT for CNV. We believe that subthreshold TTT could be a viable method of selectively confining the effect of laser on pigment epithelium, thus stimulating recovery of the outer blood-retinal barrier once the pump function has been regained. Conversely, this method may also have an impact on choroidal hyperpermeability, because retinochoroidal temperature elevation is also associated with heat shock protein (HSP) hyperexpression, producing a dual effect on the RPE/choroid complex. ${ }^{32,33}$ The hypothesis is that confined, moderate subthreshold thermal elevation at the level of the RPE/choroid complex may stimulate prompt repair of the RPE with HSP-induced cellular protection, in addition to reducing and accelerating functional recovery of choroidal cells.

Pigmentary changes, RPE mottling, or loss of transparency were not visible at ophthalmoscopy or during administration of FA and ICGA or throughout patient follow-up. We do agree that long-term follow-up is needed to examine maintenance of treatment gains and side effects. One of the seven eyes did not show any improvement in VA, revealing reduced/ persistent neurosensory detachment at OCT. Although we are unable to provide a clear explanation for this, the long-term duration of the disease may have a negative influence on retinal recovery due to atrophic changes in the outer retinal layer (outer plexiform layer and RPE) and the irreversible death of photoreceptors.

In conclusion, although this is a pilot study with several obvious limitations related to the inherent methodology in the study design, the lack of matched controls, the limited follow-up, and the number of patients, the treated eyes did show anatomic and functional improvements. This report is proof of the concept that TTT is at least effective in the short term in the treatment of serous detachment associated with CCSC. 


\section{Disclosure}

The authors report no conflicts of interest in this work.

\section{References}

1. Gass JDM. Pathogenesis of disciform detachment of the neuroepithelium: II Idiopathic central serous choriodopathy. Am J Ophthalmol. 1967;63(3): Suppl:1-139.

2. Schatz H, Madeira D, Johnson RN, McDonald HR. Central serous chorioretinopathy occurring in patients 60 years of age and older. Ophthalmology. 1992;99(1):63-67.

3. Ciardella AP, Guyer DR, Spitznas M, Yannuzzi LA. Central serous chorioretinopathy. Retina. 3rd ed. St Louis: Mosby; 2001:1153-1181.

4. Gass JDM. Stereoscopic Atlas of Macular Diseases: Diagnosis and Treatment. 4th ed. St Louis: Mosby; 1997:52-70.

5. Hussain D, Gass JD. Idiopathic central serous chorioretinopathy. Indian J Ophthalmol. 1998;46(3):131-137.

6. Lafaut BA, Salati C, Priem H, De Laey JJ. Indocyanine green angiography is of value for the diagnosis of chronic serous chorioretinopathy in elderly patients. Graefes Arch Clin Exp Ophthalmol. 1998;236(7): 513-521.

7. Prunte C, Flammer J. Choroidal capillary and venous congestion in central serous chorioretinopathy. Am J Ophthalmol. 1996;121(1):26-34.

8. Yap EY, Robertson DM. Long-term outcome of central serous chorioretinopathy. Arch Ophthalmol. 1996;114(6):689-692.

9. Schatz H, Madeira D, Johnson RN, McDonald HR. Central serous chorioretinopathy occurring in patients 60 years of age and older. Ophthalmology. 1992;99(1):63-67.

10. Klein ML, van Burskirk EM, Freidman E, et al. Experiences with non-treatment of central serous chorioretinopathy. Arch Ophthalmol. 1974;91:247-250.

11. Bujarborua D. Long-term follow-up of idiopathic central serous chorioretinopathy without laser. Acta Ophthalmol Scand. 2001;79(4): 417-421.

12. Gilbert CM, Owens SL, Smith PD, Fine SL. Long-term follow-up of central serous chorioretinopathy. Br J Ophthalmol. 1984;68(11):815-820.

13. Robertson DM, Ilstrup D. Direct, indirect and sham laser photocoagulation in the management of central serous chorioretinopathy. Am J Ophthalmol. 1983;95(4):457-466.

14. Yannuzzi LA, Slakter JS, Kaufman SR, Gupta K. Laser treatment of diffuse retinal pigment epitheliopathy. Eur J Ophthalmol. 1992;2(3): 103-114.

15. Parodi MB, Pozzo SDA, Ravalico G. Photodynamic therapy in chronic central serous chorioretinopathy. Retina. 2003;23(2):235-237.

16. Miller JW, Schmidt-Erfurth U, Sickenberg M, et al. Photodynamic therapy with verteporfin for choroidal neovascularization caused by age-related macular degeneration: results of a single treatment in a phase 1 and 2 study. Arch Ophthalmol. 1999;117(9):1161-1173.

17. Ruiz-Moreno JM, Lugo FL, Armadá F, et al. Photodynamic therapy for chronic central serous chorioretinopathy. Acta Ophthalmol. 2009;88(3): $371-376$.
18. Lee PY, Kim KS, Lee WK. Severe choroidal ischemia following photodynamic therapy for pigment epithelial detachment and chronic central serous chorioretinopathy. Jpn J Ophthalmol. 2009;53(1): 52-56.

19. Yaman A, Arikan G, Saatci AO, Cingil G. Choroidal neovascularization following photodynamic therapy in a patient with chronic central serous chorioretinopathy. Bull Soc Belge Ophtalmol. 2007;(303):69-73.

20. Myint K, Armbrecht AM, Mon S, Dhillon B. Transpupillary thermotherapy for the treatment of occult $\mathrm{CNV}$ in age-related macular degeneration: a prospective randomized controlled pilot study. Acta Ophthalmol Scand. 2006;84(3):328-332.

21. Parrozzani R, Boccassini B, De Belvis V, et al. Long-term outcome of transpupillary thermotherapy as primary treatment of selected choroidal melanoma. Acta Ophthalmol. 2008;87(7):789-792.

22. Forte R, Cennamo G. Transpupillary thermotherapy of choroidal melanomas. J Fr Ophtalmol. 2008;31(3):279-281.

23. Vianna RN, Fernandes L, Muralha A, et al. Transpupillary thermotherapy in the treatment of circumscribed choroidal hemangiomas. Int Ophthalmol. 2004;25(2):117-121.

24. Hussain N, Khanna R, Hussain A, Das T. Transpupillary thermotherapy for chronic central serous chorioretinopathy. Graefes Arch Clin Exp Ophthalmol. 2006;244(8):1045-1051.

25. Shukla D, Kolluru C, Vignesh TP, et al. Transpupillary thermotherapy for subfoveal leaks in central serous chorioretinopathy. Eye. 2008; 22(1):100-106.

26. Spitznas M. Pathogenesis of central serous retinopathy: a new working hypothesis. Graefes Arch Clin Exp Ophthalmol. 1986;224(4): 321-324.

27. Marmor MF. New hypotheses on the pathogenesis and treatment of serous retinal detachment. Graefes Arch Clin Exp Ophthalmol. 1988; 226(6):548-552.

28. Yao XY, Marmor MF. Induction of serous retinal detachment in rabbit eyes by pigment epithelial and choriocapillary injury. Arch Ophthalmol. 1992;110(4):541-546.

29. Dohrmann J, Lommatzsch A, Spital G, Pauleikhoff D. Pathogenesis of central serous chorioretinopathy: angiographic and electrophysiological studies. Ophthalmologe. 2001;98(11):1069-1073.

30. Reichel E, Berrocal AM, Ip M, et al. Transpupillary thermotherapy of occult subfoveal choroidal neovascularization in patients with age-related macular degeneration. Ophthalmology. 1999;106(10): 1908-1914.

31. Ming Y, Algvere PV, Odergren A, et al. Subthreshold transpupillary thermotherapy reduces experimental choroidal neovascularization in the mouse without collateral damage to the neural retina. Invest Ophthalmol Vis Sci. 2004;45(6):1969-1974.

32. Desmettre T, Maurage CA, Mordon S. Transpupillary thermotherapy (TTT) with short duration laser exposures induce heat shock protein (HSP) hyperexpression on choroidoretinal layers. Lasers Surg Med. 2003;33(2):102-107.

33. Desmettre T, Maurage CA, Mordon S. Heat shock protein hyperexpression on chorioretinal layers after transpupillary thermotherapy. Invest Ophthalmol Vis Sci. 2001;42(12):2976-2980.
Clinical Ophthalmology

\section{Publish your work in this journal}

Clinical Ophthalmology is an international, peer-reviewed journal covering all subspecialties within ophthalmology. Key topics include: Optometry; Visual science; Pharmacology and drug therapy in eye diseases; Basic Sciences; Primary and Secondary eye care; Patient Safety and Quality of Care Improvements. This journal is indexed on PubMed Submit your manuscript here: http://www.dovepress.com/clinical-ophthalmology-journal

\section{Dovepress}

Central and CAS, and is the official journal of The Society of Clinical Ophthalmology (SCO). The manuscript management system is completely online and includes a very quick and fair peer-review system, which is all easy to use. Visit http://www.dovepress.com/testimonials. php to read real quotes from published authors. 